\title{
Painless jaundice, pruritus
}

\begin{abstract}
Of all the GI malignancies, pancreatic adenocarcinoma is the second most common cause of death from cancer. In general it is a malignancy of the elderly with vast majority of cases occurring after the age of 60 . Due to usually advanced stage at the time of a diagnosis it remains a disease with very poor prognosis and high morbidity. Approximately two thirds of all pancreatic carcinomas occur within the head or neck of the pancreas. Pancreatic cancer is notoriously difficult to diagnose in its early stages. "Early" pancreatic cancer may be defined based on respectability, size or curability. Detecting resectable cancer is the first step in the fight against pancreatic cancer. "Early" pancreatic cancer defined as tumors $\leq 20$ $\mathrm{mm}$ in size is also called "small" pancreatic cancer. The most stringent definition of "early" pancreatic cancer is that of curable pancreatic cancer. The classical direct tumour sign is the presence of a hypoattenuating/hypointense mass at contrast-enhanced MDCT or MRI. There are also six indirect tumour signs which should suggest the possibility of a pancreatic mass: the presence of (1) biliary duct dilatation, (2) pancreatic duct dilatation, or a (3) double duct sign, which may lead to (4) focal atrophy of the gland. If a mass is sufficiently large it will (5) distort the contour of the gland, and (6) loss of pancreatic lobulation.
\end{abstract}

Volume 5 Issue I - 2016

\section{Radevic Aleksandar}

Head of the Department of Body Imaging MRI, KCS Belgrade, Serbia

Correspondence: Radevic Aleksandar, Assistant Director of the Centre for Radiology and MRI, Head of the Department of Body Imaging MRI, KCS Belgrade, Serbia,

Email voodoomdrs@yahoo.com

Received: April 28, 2016 | Published: June 15, 2016

\section{Case history}

37 years old woman with a known history of a biliary calculus presented in radiology department of a Centre of Emergency Medicine, with a painless jaundice and a mild pruritus. She was referred to the radiology department by the surgeon on call with the clinical diagnosis of a biliary colica.

US examination revealed a mildly distended gallbladder with a calculus measuring $15 \times 10 \mathrm{~mm}$ in the distal third of the gallbladder lumen, without the US signs of the inflammation of a gallbladder wall. A dilatation of intra and ekstrahepatic biliary ducts as well as a dilatation of a common pancreatic duct was noted, producing the so called" double duct sign". A fusiform aneurysm of a distalsuprabifurcational- segment of the abdominal aorta was also visualized.

A hypoechogenic than adjacent pancreatic tissue around mass lesion was visualized in the distal part of the pancreatic head-proximal part of uncinatus of the pancreas. The rest of pancreas parenchima was unchanged. Because of the presence of the direct tumour sign-the presence of a hypoechogenic mass in the distal part of the pancreatic head-proximal part of processus uncinatus of the pancreas and three of indirect tumour signs which should suggest the possibility of a pancreatic mass: the presence of ${ }^{1}$ biliary duct dilatation, ${ }^{2}$ pancreatic duct dilatation, ${ }^{3}$ and a double duct sign, the radiologist concluded that there is high probability of a pancreatic neoplasm and ordered a MSCT scan of the abdomen.

MSCT scan was performed unenhanced and on a on dynamic contrast-enhanced MDCT study with a volume of contrast medium $(2 \mathrm{~mL} / \mathrm{kg} \mathrm{b.w}$ ) is given by rapid infusion (flow rate $4 \mathrm{~mL} / \mathrm{s}$ ) and MDCT is used in the parenchymal phase, with an imaging delay of aortic transit time plus $25 \mathrm{~s}$, and in the venous phase (Figures 1-5). The cutoff of a distal segment of CBD and a dilatation of a CPD due to lateral compression from the pancreatic mass. The mass measured $16 \times 10 \times 17 \mathrm{~mm}$, without the infiltration of peripancreatic space. No distant metastases in the abdomen were seen, and no enlarged lymph nodes were noted.

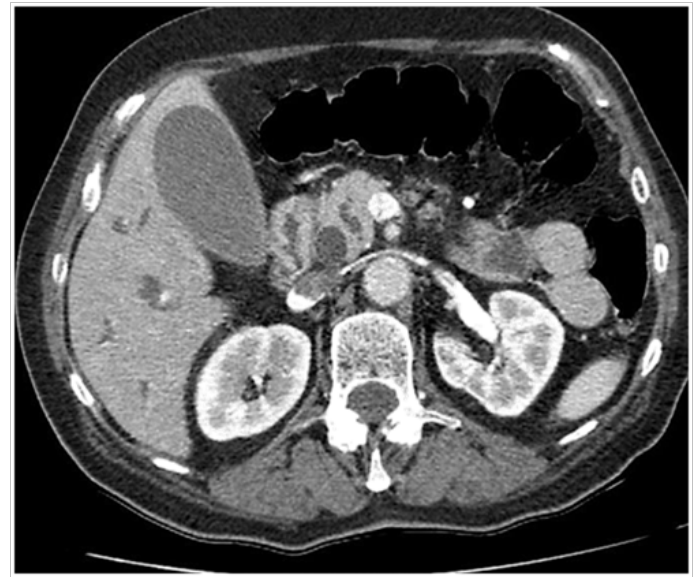

Figure I Axial MDCT shows a double duct sign in pancreatic cancer.

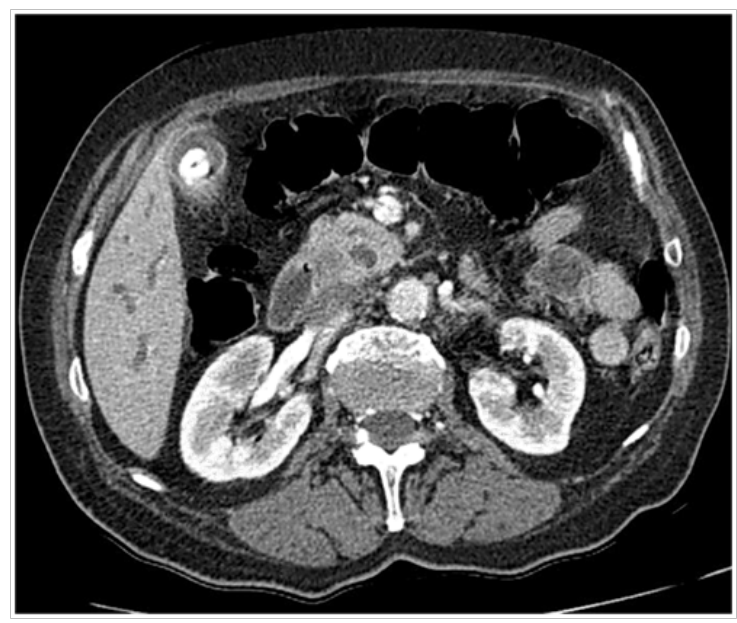

Figure 2 Axial MDCT shows a hypoattenuating mass in the head and uncinate process of the pancreas with a dilatation of the pancreatic duct and the bile duct, and a calculus in the distal third of the gallbladder lumen. 


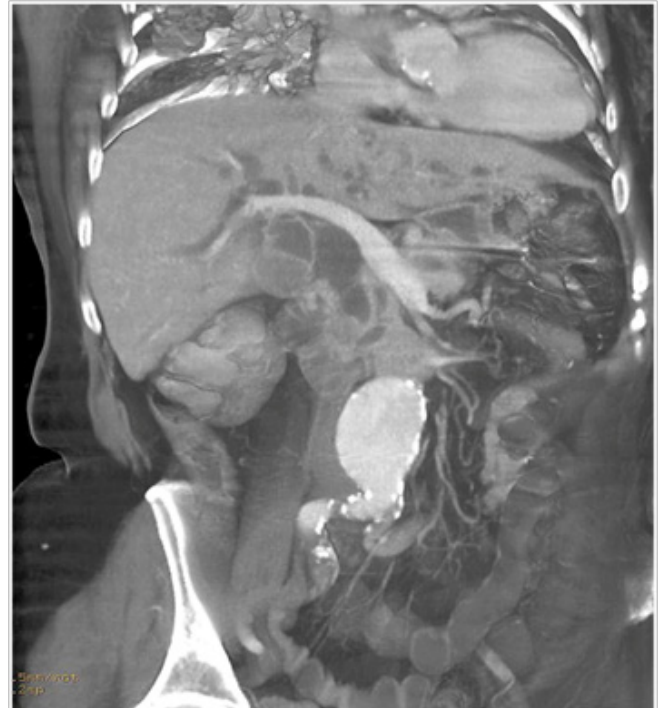

Figure 3 Curved planar reformation shows the mass in the head of the pancreas with pancreatic duct obstruction due to the compression of its lateral wall, CBD dilatation with a curvilinear cut off sign. Fusiform aneurism of the suprabifurcational segment of the abdominal aorta with linear calcifications of the aortic wall.
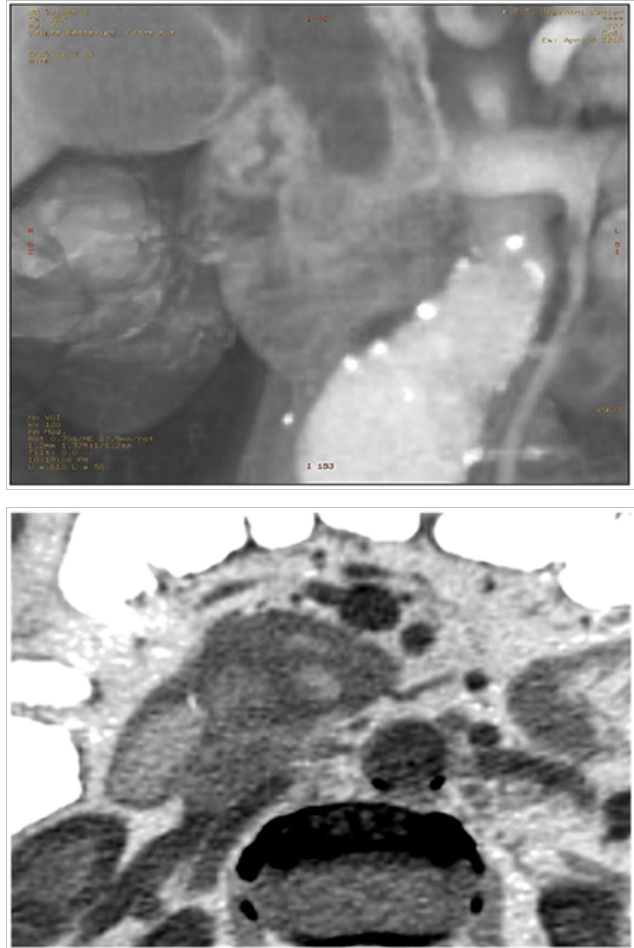

Figure $\mathbf{4}$ Close up views of a hypoattenuating mass in the head and uncinate process of the pancreas with relatively distinct borders of the hypoattenuating mass to the surrounding pancreatic tissue.

\section{Diagnosis}

a. Histology proven Adenocarcinoma of the head of the pancreas, without local lymph node infiltration/all extirpated lymph nodes were cancer free.

b. This was diagnosed as T1M0N0 or T1Bb stage Adnocarcinoma of the head of the pancreas.

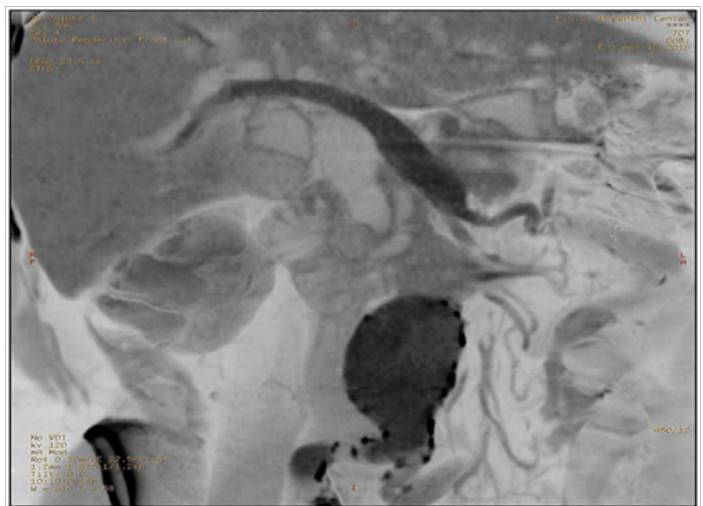

Figure 5 Curved planar reformation inverse gray shows more clearly the mass in the head of the pancreas.

\section{Findings}

In a patient with a painless jaundice on the US examination of the abdomen a dilated intra and extra hepatic bile ducts, dilated CPD were found as well as hypoechogenic focal lesion in the distal part of the head- processus uncinatus of the pancreas. Abdominal fusiform aneurysm was also visualized MSCT of the abdomen, unenhanced and dynamic contrast-enhanced MDCT study showed a hypoattenuating mass in the head and uncinate process of the pancreas measuring $16 \times 10 \times 17 \mathrm{~mm}$ with a dilatation of the pancreatic duct and the bile duct -the double duct sign and a calculus in the distal third of the gallbladder lumen. No enlarged lymph nodes. No distant metastases

\section{Differential diagnosis}

a. Pancreatic head carcinoma -Chronic pancreatitis -Groove pancreatitis.

b. Pancreatic head carcinoma On CT the mass may not be visible on a preliminary unenhanced image but, because the tumour is typically less vascular than the surrounding normal pancreatic parenchyma, it will be seen as a poorly enhancing focal area within the densely enhancing normal pancreatic tissue on dynamic contrast-enhanced MDCT. Tumours in the head of the pancreas may cause early dilatation of the common bile duct, although masses arising in the uncinate process are often quite large, encasing the superior mesenteric artery before bile duct obstruction occurs.

c. Chronic pancreatitis (CP) is an irreversible inflammatory disease of the pancreas, resulting in fibrosis. When duct dilatation accompanies focal enlargement of the gland, the appearance may mimic that of pancreatic carcinoma. Chronic pancreatitis may present with a focal mass, thus mimicking ductal adenocarcinoma

d. Groove pancreatitis is a distinct form of $\mathrm{CP}$, affecting the groove between the pancreatic head, duodenum and common bile duct. This may lead to pancreatic and/or bile duct dilatation.

\section{Discussion}

Approximately $70 \%$ of adenocarcinomas arise in the head or uncinate process, with the remainder arising in the body or tail. On US the tumour typically has a lower echogenicity than adjacent pancreatic tissue. On CT the tumour is typically less vascular than the surrounding normal pancreatic parenchyma; it will be seen as a poorly enhancing focal area within the densely enhancing normal pancreatic 
tissue on dynamic contrast-enhanced MDCT 1.Tumours in the head of the pancreas may cause early dilatation of the common bile duct and the common pancreatic duct.

For detection of pancreatic cancer, there are direct and indirect tumour signs. The classical direct tumour sign is the presence of a hypoechogenic mass at US or hypo attenuating/hypointense mass at contrast-enhanced MDCT or MRI. There are also six indirect tumour signs which should suggest the possibility of a pancreatic mass:
a. The presence of biliary duct dilatation,
b. Pancreatic duct dilatation,
c. Double duct sign-the presence of a biliary and pancreatic duct dilatation, which may lead to focal atrophy of the gland,
d. If a mass is sufficiently large it will,
e. Distort the contour of the gland,
f. Loss of pancreatic lobulation is also often an early sign 3 .

\section{Acknowledgments}

None.

\section{Conflicts of interest}

Author declares that there is no conflict of interest.

\section{References}

1. Schima W, Ba Ssalamah A, Kolblinger C, et al. Pancreatic adenocarcinoma. European Radiology. 2007;17(3):638-649.

2. Triantopoulou C, Dervenis C, Giannakou N, et al. Groove pancreatitis: a diagnostic challenge. Eur Radiol. 2009;19(7):1736-1743.

3. Takeshita K, Kutomi K, Haruyama T, et al. Imaging of early pancreatic cancer on multidetector row helical computed tomography. Br J Radiol. 2010;83(994):823-830. 